Joaquín F Mould Quevedo'

Guillermo Salinas Escudero"

Iris Contreras Hernández'

Carlos Garrido Solano"'I
Unidad de Investigación en Economía de la Salud. Instituto Mexicano del Seguro Social (IMSS). México DF, México

Dirección de Investigación. Hospital Infantil de México Federico Gómez. Secretaría de Salud. México DF, México

III División de Sistemas de Salud. Coordinación de Políticas de Salud. IMSS. México DF, México

Correspondencia | Correspondence: Guillermo Salinas Escudero

Hospital Infantil de México Federico Gómez

Dirección de Investigación

Dr. Márquez \# 162 - Colonia Doctores,

C.P. 06720. México D.F.

E-mail: gsalinas@himfg.edu.mx

\section{Competencia en el sector salud: análisis de la reforma sueca (1992 - 1995)}

\section{Competitiveness in the health care sector: analysis of the Swedish reform (1992-1995)}

\section{RESUMEN}

El artículo revisa los éxitos y fracasos de la reforma sueca de salud, así como las lecciones que dejó en su afán de alcanzar mejores resultados financieros y estándares de calidad.

DESCRIPTORES: Reforma en Atención de la Salud. Sistemas Locales de Salud. Economía de la Salud. Administración de los Servicios de Salud. Suecia.

\section{ABSTRACT}

The paper reviews the outcomes and failures of the Swedish health care reform, as well as the lessons learned for accomplishing better financial results and quality standards.

DESCRIPTORS: Health Care Reform. Local Health Systems. Health Economics. Health Services Administration. Sweden. 


\section{INTRODUCCIÓN}

La forma de generar competencia en el campo de la salud es variable en el mundo. Por lo general, toda estrategia que aspire a mejorar la competitividad de un sistema nacional de salud debe fomentar la eficiencia, la efectividad, el equilibrio económico y la calidad de la atención médica. La competencia en el campo de la salud brinda la oportunidad de mejorar de forma costo - efectiva la provisión de los servicios médicos. Asimismo, la competencia genera mayores incentivos financieros así como colabora en la mejora de la calidad de la atención. Sin embargo, no siempre se obtienen los resultados esperados, por lo que esta discusión actualmente sigue siendo un tema muy controversial. En algunos casos la competencia puede generar inequidad en el acceso a los servicios de salud ${ }^{25} \mathrm{o}$ en otros, realmente no ha mostrado ser una herramienta útil en la reducción de los costos de la atención médica en distintos países. ${ }^{21}$

Las reformas en el área de la salud orientadas al mercado realizadas en varios países desarrollados en la década de los 1980's y 1990's tuvieron varios componentes en común. Generalmente se basaron dentro de algunas de las siguientes medidas: la aplicación de ciertos pagos por parte de los usuarios para cubrir un porcentaje de los costos generados en su atención (sistemas de co-pagos), la separación entre la parte prestadora de los servicios de salud y la parte financiera, una reforma hacia un manejo gerencial de los hospitales, generación de una mayor competencia dentro de la parte proveedora y la generación de sistemas de prepago de servicios de salud mediante seguros médicos. ${ }^{19}$

El sistema de salud de Suecia es un sistema descentralizado, que antes de 1970 con la excepción de la creación de las regiones médicas de 1958 (siete regiones), pocos cambios mayores había sufrido su estructura desde que se transfirió la administración del cuidado de la salud a los consejos de los condados en 1864. En 1983 finalizó el periodo formal de descentralización desarrollado por el Estado Sueco. ${ }^{a}$

El Ministerio de Salud y Asuntos Sociales era el encargado del primer nivel de atención, del desarrollo de guías de cuidado de la salud, de servicios salud comunitarios y el aseguramiento de salud. El segundo nivel de atención estaba conformado por una serie de agencias administrativas independientes que eran supervisadas por el Consejo Nacional de Salud y Bienestar. Las siete regiones suecas de atención médica estaban conformadas en promedio por tres condados, los cuales en algunos casos compartían uno o más hospitales regionales, mismos que dependían de los consejos de los condados para la determinación del presupuesto y los servicios que ahí se ofrecían. ${ }^{\mathrm{a}}$
Entre 1992 y 1995 una nueva forma de financiar y organizar la atención médica se estableció en Suecia, el modelo Estocolmo (Stockholm model ó Swedish model), que consistía en que el rol de los compradores (distritos de salud) y los proveedores (los hospitales) estuviese separado; y que los hospitales y demás servicios de atención ambulatoria compitan entre sí para atraer nuevos pacientes, creando un mercado competitivo interno para los servicios hospitalarios. El propósito de la reforma sueca fue la de mejorar la utilización de los recursos financieros y fortalecer la posición del paciente dentro del sistema sueco para la salud (que el dinero siga al paciente). Sin embargo, habría que preguntarse ¿cuáles fueron los éxitos y fracasos de la reforma sueca? ¿Realmente impulsó la competencia dentro del sistema de salud en Suecia?

El objetivo de la presente investigación buscó analizar una de las principales reformas de salud a nivel mundial que se produjo durante los años 1990, tomada como ejemplo en muchas economías del mundo.

\section{ANTECEDENTES}

La población de Suecia es de aproximadamente de 8.9 millones de personas, en el año 2000. ${ }^{24}$ Como muchos otros países industrializados, Suecia tiene una baja tasa de natalidad (1.5 por mujer en edad reproductiva), con una esperanza de vida al nacer de 77.5 años para hombres y 81.1 años para las mujeres en el año 2000, y es el país con la mayor población de adultos mayores de 65 años en el mundo (aproximadamente un 20\%). ${ }^{16,27}$

A partir de 1990, se observó en el país una reducción significativa de la mortalidad infantil (pasó en 1990 de 6 a 3.2 muertes por cada 1,000 nacimientos en el año 2000). Las muertes por eventos cardiovasculares en el país se redujeron significativamente entre 1987 y $1997 .{ }^{27}$ En Suecia el número relativo de camas de atención hospitalaria ha venido reduciéndose significativamente, en 1973 era de 14.4 por cada 1,000 habitantes y en 1990 se redujo a 11.4 por cada 1,000 habitantes. ${ }^{16,27}$

El gasto total en salud en Suecia durante el año 2002 fue de $8.4 \%$ como porcentaje del Producto Interno Bruto (PIB) (alrededor de US\$1,746 per capita), ${ }^{27}$ del cual el $90 \%$ era realizado por el Estado. Cabe señalar que sólo un $8 \%$ de los médicos suecos trabajan a tiempo completo en el sector privado. Asimismo, el gasto público en salud se redujo de $89.9 \%$ en 1990 a $83.8 \%$ en 1998 como porcentaje del gasto total en salud, como consecuencia de las reformas iniciadas durante esta década. La Tabla 1 presenta las tendencias de gastos con salud en Suecia entre 1980 y 1998.

a Jonsson E, Banta HD. Health care technology in Sweden. In: US Congress. Office of Technology Assessment. Health care technology and its assessment in eight countries. Washington; DC; 1995. p 209-6. 
Tabla 1. Tendencias del gasto en salud en Suecia (1980 - 1998).

\begin{tabular}{|c|c|c|c|c|c|c|c|c|c|c|c|}
\hline Variable & 1980 & 1985 & 1990 & 1991 & 1992 & 1993 & 1994 & 1995 & 1996 & 1997 & 1998 \\
\hline $\begin{array}{l}\text { Gasto total en atención médica (billones de } \\
\text { euros*) }\end{array}$ & 8,5 & 12,0 & 15,9 & 16,7 & 16,9 & 14,2 & 14,3 & 14,9 & 17,3 & 17,1 & 16,9 \\
\hline $\begin{array}{l}\text { Miles de Coronas* suecas/ per capita (1995 } \\
\text { - precios PIB) }\end{array}$ & 14,7 & 15,6 & 17,3 & 16,8 & 16,5 & 15,9 & 15,6 & 15,8 & 16,8 & 17,1 & 17,5 \\
\hline Participación en el PIB (\%) & 9,4 & 9,0 & 8,8 & 8,7 & 8,8 & 8,9 & 8,6 & 8,4 & 8,7 & 8,5 & 8,4 \\
\hline Gasto público como \% del gasto total en salud & 92,5 & 90,4 & 89,9 & 88,2 & 87,2 & 85,7 & 85,2 & 85,2 & 84,8 & 84,3 & 83,8 \\
\hline
\end{tabular}

Fuente: Hjortsberg \& Ghatnekar ${ }^{16}$ (2001).

* Tipo de cambio: Coronas suecas/Euros $=8,9288$.

\section{FINANCIAMIENTO DEL SISTEMA SUECO DE SALUD}

El financiamiento de la salud en Suecia se encuentra descentralizado y se genera principalmente por medio de los impuestos locales que impone cada condado, así como de otras partidas suplementarias que reciben del gobierno nacional. El gasto en salud que realizan los Condados alcanza, en promedio, un $85 \%$ de su presupuesto total. De este gasto en salud, el $66 \%$ proviene de los impuestos recolectados por cada condado y entre el $7 \%-11 \%$ proviene de los impuestos proporcionados por el gobierno central. ${ }^{20}$ Durante los últimos diez años el sistema de financiamiento en Suecia ha sufrido sólo cambios relativos. Por ejemplo, en 1992, el $70 \%$ del gasto en salud provenía de los impuestos locales (condados), el 19\% de las transferencias gubernamentales, el $3 \%$ de pagos directos de pacientes y un $8 \%$ de otros ingresos. ${ }^{\text {a }}$

En 1999, la atención de segundo y tercer nivel representaban alrededor del $62.3 \%$ del presupuesto de los condados destinados a salud, el $22.4 \%$ a la atención primaria, 9.5\% a la atención psiquiátrica y el restante $5.8 \%$ se gastaba en la atención geriátrica. Por otro lado, los médicos del sistema público no reciben dinero directamente de los pacientes sino que reciben un salario mensual de parte del gobierno de acuerdo a sus capacidades y a su programa de servicios. En contraposición, los médicos en el sector privado pueden recibir pagos directamente de los pacientes y además, si estos tienen un contrato con el condado de su localidad pueden recibir un reembolso público.

En el año 2000, los empleados públicos y privados contribuían al sistema de seguridad social con el $8.5 \%$ de su salario, en tanto que aquellos trabajadores independientes contribuían con un $8.2 \%$ de sus ingresos. Finalmente, sólo el $0.5 \%$ de la población sueca tiene un seguro de salud privado (alrededor de 120,000 personas) el resto de personas se encuentra asegurada por la seguridad social. ${ }^{27}$

\section{¿POR QUÉ SE REFORMÓ EL SISTEMA DE SALUD EN SUECIA?}

El poder económico de Suecia, medido por el PIB per cápita, ha venido disminuyendo desde $1970 .{ }^{12}$ Como consecuencia de la recesión económica a comienzos de los 1990 y las largas listas de espera que registraba la atención médica en dichos años, se decidió cambiar la forma de financiar y organizar la provisión de los servicios de salud. Es en este ambiente, donde se decidió iniciar el pago prospectivo a los hospitales con el propósito de impulsar la productividad del sector.

En un estudio para los países de la Organization for Economic Co-Operation and Development (OECD), Fare et $\mathrm{al}^{9}$ encontró un crecimiento en la productividad durante el período comprendido entre 1974 y 1989. Los dos países que registraron el mayor crecimiento acumulado fueron Dinamarca (+15\%) y Estados Unidos (+5\%), siendo la razón principal en ambos países de dicho crecimiento las mejoras tecnológicas acontecidas en el sector. No obstante, en Suecia existía una alta necesidad de mejorar la productividad de la atención médica así como mejorar el control de los gastos debido al bajo crecimiento económico que había sufrido este país desde los años $1970 .{ }^{12}$

A comienzos de los años 1990 muchos condados en Suecia inició el pago prospectivo de sus hospitales por medio de un monto fijo por servicios brindados (utilizando los Diagnosis Related Groups -DRGs, hospitalarios y algunos no hospitalarios). La iniciativa de emplear DRG nació en 1985 por medio del Swedish Institute for Health Services Development (SPRI). En 1987, se realizó en Suecia el primer proyecto a nivel clínico utilizando DRG. Seguidamente, entre 1989 y 1992 se realizó un segundo proyecto utilizando DRG para probar el sistema de reembolso de costos en el hospital Helsingborg. En base a estos proyectos se determinó que el sistema de DRG sueco no podía utilizar los pesos norteamericanos. ${ }^{b}$

\footnotetext{
a Smith P. Capitated finance and health care needs: possibilities and limitations. In: 19. Jornadas de Economía de la Salud; 1999 jun 2-4;

Zaragoza, España. Huesca: Asociación de Economía de la Salud; 1999. p. 15-28.

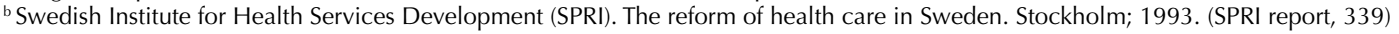


Las razones por las que se reformó el sistema de salud en Suecia son muchas. ${ }^{12}$ Una de ellas tiene que ver con la caída de la productividad en Suecia durante 1960 -1980, con media de 3\% al año, lo cuál significaba que el costo de la atención médica en términos reales fuese en 1980 el doble de lo que fue en 1960. Por tal razón, esto condujo a que se buscaran otras medidas para mejorar la productividad como lo fueron los DRG. Otro factor que impulsó las reformas en Suecia fue el análisis del sistema de salud sueco que realizó Enthoven, donde se sugirió la separación entre el proveedor de los servicios y su comprador, tal como refería en la nueva manera de organizar y financiar la atención médica. ${ }^{8}$ Adicionalmente, factores como el crecimiento económico negativo por tres años consecutivos (1991 - 1993) promovieron el cambio en el sistema de salud sueco. Aún así Suecia mostraba antes de las reformas uno de los niveles de satisfacción en usuarios de la atención médica más altos de Europa en relación con el gasto en salud per capita (68\% en 1996). ${ }^{23}$

\section{LA REFORMA SUECA (1992 - 1995)}

Entre 1992 y 1995 una nueva forma de financiar y organizar la atención médica se estableció en Suecia. ${ }^{5}$ La nueva reforma sueca basada en una forma original de transferir costos consistía principalmente en un contrato basado en pagos prospectivos por caso (per case) empleando la metodología de los DRG complementado con garantías para preservar la calidad de la atención médica. Las estancias hospitalarias en Suecia serían pagadas por un pago fijo prospectivo extraído de un precio de lista según los DRG. En el caso de la atención ambulatoria, los pesos de las visitas se obtuvieron de un programa de pago por caso generado localmente. Adicionalmente, se emplearon pagos per diem (promedio) como complemento del pago por caso dependiendo de la complejidad de los pacientes (por ejemplo, en aquellos casos donde los DRG no capturaban todos los costos). ${ }^{5}$

El pago prospectivo por caso variaba entre los distintos condados y hospitales. En Suecia llegó a existir paralelamente tres tipos de DRG, DRG - Health Care Financing Administration (HCFA's - DRG), All-Patient DRG (AP-DRG) y Refined DRG (R-DRG). Nunca hubo ningún esfuerzo gubernamental por uniformizarlos a todos en uno solo en la experiencia sueca. Sin embargo, 14 de los 24 condados que existían en 1992 continuaron con el pago tradicional (financiamiento de sus hospitales con un presupuesto global anual fijo). La razón principal por la que no todos los condados en Suecia adoptaron la reforma parte de que mucho se asoció este nuevo mecanismo con reducciones en los presupuestos, lo cuál en muchos condados generó un malestar anticipado. De esta forma, el sistema de salud sueco es una mezcla de pagos prospectivos por caso y presupuestos globales anuales fijos.

La Tabla 2 presenta los modelos de financiamiento y forma de pago por atención hospitalaria en Europa.

El modelo sueco funcionaba de la siguiente manera: los distritos locales de salud compraban servicios hospitalarios en beneficio de la población según un determinado presupuesto. Los hospitales recibían en compensación un pago de los distritos de salud de acuerdo a las actividades realizadas; se emplearon los DRG como medida final de producto hospitalarios, no hospitalarios y en los casos pertinentes otras medidas cuando los servicios no eran hospitalarios. De esta forma, la separación entre el proveedor y el comprador de servicios de salud se hacia más transparente; los distritos de salud compraban los servicios de atención médica del proveedor de salud que en este caso eran los hospitales en manos de los condados. Por otro lado, los distritos de salud no compiten entre sí, ya que estos sólo compran servicios médicos para los residentes de su área; sin embargo, los pacientes no pueden modificar a las autoridades del distrito a menos que cambien de residencia.

Otro punto importante de la reforma sueca fue la posibilidad de otorgarle a los pacientes la libre elección de su médico, del centro de salud, hospital e inclusive de un médico privado financiado por el sistema público de salud. ${ }^{13}$ Esta medida reflejaba un desarrollo de la sociedad sueca en su búsqueda por alcanzar una mayor libertad para sus ciudadanos en materia pública.

En Suecia los salarios de los médicos se encontraban tradicionalmente en manos de los consejos de cada condado. Sin embargo, en este ramo, también se realizaron propuestas para mejorar la calidad y la productividad de los médicos. En algunos Condados se introdujo los pagos por capitación a los médicos del primer nivel de la atención y de urgencias en aras de compensar al área médica por la libre elección de los pacientes, así como para crear incentivos para atraer nuevos pacientes y mejorar los servicios de salud. Según Smith, ${ }^{a}$ el modelo de capitación sueco es uno de los más avanzados del mundo ya que incorpora aspectos adecuados a nivel individual entre los pacientes. Con ello se buscaba suplir la ausencia de incentivos que existen en todo sistema asalariado, así como incrementar la carga de trabajo (productividad). Debe señalarse que a la oferta pública de la atención ambulatoria tradicional se sumaron médicos que operan en la iniciativa privada previo acuerdo con los condados. Finalmente, la reforma fue complementada en 1993, al fijarse principios éticos a todo el conjunto de medidas, tales como delimitar principios para evitar discriminaciones basadas en la edad de los pacientes o su status socioeconómico.

a Smith P. Capitated finance and health care needs: possibilities and limitations. In: 19. Jornadas de Economía de la Salud; 1999 jun 2-4; Zaragoza, España. Huesca: Asociación de Economía de la Salud; 1999. p. 15-28. 
Tabla 2. Financiamiento y forma de pago por atención hospitalaria en Europa.

\begin{tabular}{|c|c|c|c|}
\hline \multirow[t]{2}{*}{ País } & \multirow{2}{*}{$\begin{array}{l}\text { Fuente de financiamiento } \\
\text { principal para los servicios } \\
\text { de atención hospitalaria }\end{array}$} & \multicolumn{2}{|c|}{$\begin{array}{l}\text { Principal aproximación para el pago de los costos } \\
\text { en los servicios hospitalarios agudos }\end{array}$} \\
\hline & & Financiación prospectiva & Servicios basados en el financiamiento \\
\hline Austria & $\begin{array}{l}\text { Seguro Social de } \\
\text { Financiamiento }\end{array}$ & NA & $\begin{array}{l}\text { Seguro Social de Financiamiento basado en los } \\
\text { días de estancia hospitalaria con un impuesto } \\
\text { de suma fija por el Ministerio de Salud }\end{array}$ \\
\hline Dinamarca & $\begin{array}{l}\text { Descentralizado, } \\
\text { financiado a través } \\
\text { de impuestos }\end{array}$ & $\begin{array}{l}\text { Presupuestos globales } \\
\text { prospectivos }\end{array}$ & NA \\
\hline Inglaterra & $\begin{array}{l}\text { Centralizado, financiado } \\
\text { a través de impuestos }\end{array}$ & NA & $\begin{array}{l}\text { El pago se encuentra determinado por } \\
\text { los contratos comprador/proveedor. }\end{array}$ \\
\hline Finlandia & $\begin{array}{l}\text { Descentralizado, } \\
\text { financiado a través } \\
\text { de impuestos }\end{array}$ & NA & $\begin{array}{l}\text { Los pagos por los servicios son } \\
\text { reintegrados por la municipalidad. }\end{array}$ \\
\hline Francia & $\begin{array}{l}\text { Seguro Social de } \\
\text { Financiamiento }\end{array}$ & $\begin{array}{l}\text { Presupuestos globales } \\
\text { prospectivos }\end{array}$ & NA \\
\hline Alemania & $\begin{array}{l}\text { Seguro Social de } \\
\text { Financiamiento }\end{array}$ & $\begin{array}{l}\text { Presupuestos flexibles } \\
\text { prospectivos }\end{array}$ & NA \\
\hline Hungría & $\begin{array}{l}\text { Seguro Social de } \\
\text { Financiamiento }\end{array}$ & NA & $\begin{array}{c}\text { El pago se encuentra asociado al } \\
\text { desempeño hospitalario basado en GRD. }\end{array}$ \\
\hline Irlanda & $\begin{array}{l}\text { Centralizado, financiado } \\
\text { a través de impuestos }\end{array}$ & $\begin{array}{l}\text { Presupuestos globales } \\
\text { prospectivos }\end{array}$ & NA \\
\hline Italia & $\begin{array}{l}\text { Centralizado, financiado } \\
\text { a través de impuestos }\end{array}$ & $\begin{array}{l}\text { Presupuestos globales } \\
\text { prospectivos }\end{array}$ & NA \\
\hline Latvia & Impuestos & NA & $\begin{array}{l}\text { Pago variable diario y financiación } \\
\text { según los servicios relacionados. }\end{array}$ \\
\hline Holanda & $\begin{array}{l}\text { Seguro Social de } \\
\text { Financiamiento }\end{array}$ & $\begin{array}{l}\text { Presupuestos funcionales } \\
\text { prospectivos basados } \\
\text { parcialmente en la actividad. }\end{array}$ & NA \\
\hline Noruega & $\begin{array}{l}\text { Descentralizado, } \\
\text { financiado a través } \\
\text { de impuestos }\end{array}$ & $\begin{array}{l}\text { Presupuestos globales } \\
\text { prospectivos }\end{array}$ & NA \\
\hline Polonia & Impuestos & Presupuestos globales anuales & NA \\
\hline Eslovaquia & Seguro Nacional de Salud & NA & Pago por día/cama de estancia hospitalaria. \\
\hline Eslovenia & Seguro Nacional de Salud & NA & $\begin{array}{l}\text { Financiamiento prospectivo anual basado en } \\
\text { contratos que incorporen el pago de día/cama } \\
\text { y otros servicios que requieran financiamiento }\end{array}$ \\
\hline Suecia & $\begin{array}{l}\text { Descentralizado, } \\
\text { financiado a través } \\
\text { de impuestos }\end{array}$ & $\begin{array}{l}\text { Prospectivo con presupuesto } \\
\text { departamental combinado } \\
\text { con el pago por actividad. }\end{array}$ & NA \\
\hline
\end{tabular}

NA: No Aplica

Fuente: WHO (1996).

\section{RESULTADOS DE LA REFORMA}

Los resultados para el primer año (1992) fueron exitosos. ${ }^{12}$ Por ejemplo, la atención hospitalaria aumentó en $8 \%$, las cirugías en un $50 \%$ y las visitas médicas ambulatorias en un $15 \%$. Tomando todas las actividades juntas el incremento de la productividad fue del $11 \%$. Asimismo, debido a que a principios de 1992 se redujo en $10 \%$ los precios de los DRG, los costos cayeron en un $1 \%$ debido también a una reducción en el personal médico. Sin embargo, en 1994 se comenzaron a registrar ya algunos problemas asociados a las reformas. También hubo un incremento importante del gasto lo cuál condujo a que en 1994 existiese un déficit presupuestal del 4\%. Esto representó que la oferta total de la atención médica que se empleaba superaba el presupuesto total. El modelo sueco tendía a incrementar significativamente la productividad de las unidades médicas. ${ }^{5,12}$

En un estudio ${ }^{18}$ se comparó cinco condados que emplearon la reforma (Dalarma, Stockholm, Bohuslän, 
Tabla 3. Porcentaje de cambio en los resultados, costos y productividad de la atención médica aguda para cinco condados que emplearon la reforma versus el sistema tradicional de presupuestos (grupo control). Suecia, 1990-1993.

\begin{tabular}{|c|c|c|c|c|c|c|}
\hline \multirow{2}{*}{ Variable } & \multirow[t]{2}{*}{ Grupo Control } & \multicolumn{5}{|c|}{ Condado } \\
\hline & & Sörmland & Stockholm & Dalarna & Bohuslän & Örebro \\
\hline Resultados & - & 9,2 & 11 & $-0,8$ & 7,2 & 7,7 \\
\hline Costos & $-3,4$ & $-4,3$ & $-4,0$ & $-11,4$ & $-8,7$ & 7,0 \\
\hline Productividad & 2,4 & 14,1 & 16 & 12,1 & 17,4 & 0,7 \\
\hline
\end{tabular}

Fuente: Jonsson ${ }^{18}$ (1996).

Nota: La celda correspondiente a Grupo Control se encuentra en blanco debido a que el grupo control al no presentar una intervención, mantiene la estructura existente de atención de egresos hospitalarios y consultas (por lo tanto el resultado no se modifica).

Sörmland y Örebro) contra 14 condados que no la emplearon (grupo control). Los resultados del estudio evidenciaron que la mayor productividad durante el período 1990-1993 se produjo en los condados que adoptaron la reforma. El incremento de la productividad en estos condados fue de $14.1 \%, 16.0 \%$, $12.1 \%, 17.4 \%$ y $0.7 \%$, respectivamente. Durante ese mismo tiempo, el grupo control sólo incrementó su productividad en $2.4 \%$. La medida de productividad utilizada en este estudio fue compuesta por medidas de atención hospitalaria y ambulatoria en relación con el costo. ${ }^{18}$

La razón por la que en el condado de Örebro tuvo una baja productividad corresponde a que en este condado los precios de lista de los DRG no se redujeron por anticipado como sucedió en Stockholm y Bohuslän. Es decir, el incremento en los costos de Örebro fue directamente proporcional al incremento del producto tal como lo muestra el Tabla 3. Sin embargo, Håkansson también señala que hubo tres condados que no adoptaron la reforma (Gävleborg, Jönköping y Värmland) y que mostraron un desempeño igual o superior al alcanzado por los cinco condados del ejemplo anterior (Tabla 4). ${ }^{18}$

Gerdtham \& Rehnberg ${ }^{10}$ evaluaron las diferencias en costos y productividad para todos los condados en Suecia entre 1992-1993. Los autores hallaron que en los condados donde se habían realizado la separación entre el comprador y el proveedor, mostraron mejoras más en la productividad y que la transferencia de costos descentralizada redujo en 13\% los costos..$^{10}$ Carpentier \& Samuelson ${ }^{6}$ señalaron que el modelo Stockholm durante el período de 1992-1997 contribuyó tanto en el corto como en el largo plazo a efectos positivos. Durante los primeros años los autores observaron un incremento sustancial de la producción y la productividad.

Los tiempos de espera disminuyeron dramáticamente. ${ }^{14,15}$ Después del primer año de iniciada la reforma, los condados que la implementaron mostraron una importante reducción en los tiempos de espera, en especial para los tratamientos hospitalarios así como algunas cirugías de cataratas y de cadera., ${ }^{413}$ También existen registros de que hubo reorganizaciones internas significativas en varios hospitales orientadas a mejorar la eficiencia. ${ }^{15}$

\section{CRITICAS AL MODELO DE COMPETENCIA SUECO}

El efecto negativo que presentó la reforma sueca de salud fue que la misma no estuvo acompañada de un desarrollo gerencial idóneo, que orientara la prestación de servicios a las necesidades de la población, de tal forma que equilibrara el incremento en la productividad con un ajuste de la capacidad y estructura que el sistema de salud requería. ${ }^{6}$ Una consecuencia de la utilización de DRG como mecanismo de pago prospectivo de servicios de salud, es que los médicos y los hospitales tienden a la especialización de los servicios prestados (incremento en la productividad) para obtener un mayor presupuesto y a la prestación de servicios de forma indiscriminada. Como resultado se impacta de forma importante en el gasto final en salud, sin que en algunos casos realmente haya un impacto en términos de la salud de las personas.

Al no existir una instancia que hiciera una evaluación entre la congruencia de los DRG reportados por lo hospitales y los requerimientos reales de atención médica de la población, conllevó de forma inherente a los hospitales a realizar una sobreutilización de los servicios. Los hospitales aprovecharan la asimetría de

Tabla 4. Porcentaje de cambio en los resultados, costos y la productividad en la atención médica aguda en condados que no adoptaram la reforma. Suecia ,1990-1993.

\begin{tabular}{lccc}
\hline Variable & \multicolumn{3}{c}{ Condado } \\
& Gävleborg & Värmland & Jönköping \\
\hline Resultados & 2,1 & 8,3 & 18,0 \\
Costos & $-12,9$ & $-7,3$ & $-4,0$ \\
Productividad & 17,2 & 16,8 & 22,9 \\
\hline
\end{tabular}

Fuente: Jonsson ${ }^{18}$ (1996). 
la información que tienen los médicos respecto a los pacientes y al área financiadora para incrementar de forma desmedida la prestación de servicios, teniendo en cuenta que tiene la posibilidad de generan su propia demanda artificial de servicios de salud. O puede ocurrir lo contrario, que los financiadores limiten excesivamente los precios de los DRG que están dispuestos a pagar. ${ }^{19}$

Como muestra del descontento público en Suecia, la satisfacción en usuarios de la atención médica cayó entre 1996 y 1998 en un 10\% (58\% en 1998), siendo una de las caídas más altas por este concepto en toda Europa. Aun cuando existen estudios que evaluaron que la calidad de la atención médica se mantuvo igual con las nuevas reformas de salud. ${ }^{\mathrm{a}}$

Si bien los condados que adoptaron la reforma fueron más productivos en el corto plazo, el sistema tradicional de pagos resultó más eficiente al momento de contener los gastos. ${ }^{3,18}$ La mayor productividad observada en los condados que implementaron la reforma tendía a elevar los costos, los cuáles al cabo de un período corto de tiempo ya excedían los presupuestos iniciales destinados para el sector. ${ }^{13}$ En este sentido, los precios de los DRG utilizados por el nuevo sistema tuvieron que ser nuevamente reducidos en 1994, y de esta manera se comenzó a cuestionar la idoneidad del sistema de pago prospectivo a los hospitales suecos. Otros autores señalan que un pago prospectivo como el empleado durante la reforma generaba serios desincentivos así como motivó una reducida preocupación en los profesionales de la salud con relación a las consecuencias sociales de las enfermedades. ${ }^{26}$

La entrada al gobierno del Partido Social Demócrata modificó la palabra competencia por cooperación. Lo mismo sucedió en otros países como en el Reino Unido o Nueva Zelanda. ${ }^{2,22}$ Lo que sucedió en Suecia fue que la introducción de la mayor competencia generó algunos monopolios locales en determinados servicios de la atención médica. Esto agravó aún más con las fuertes asimetrías de información que condujo a una ausencia de demanda lo suficientemente capacitada para hacer frente al nuevo mercado de la salud. ${ }^{22}$ La cooperación buscada sustituyó los contratos de corto por los de largo plazo entre los compradores y los proveedores de servicios de salud para el pago de la atención médica. ${ }^{4}$

De acuerdo con Ham \& Brommels, ${ }^{13}$ la mayor competencia en el sistema de salud sueco era predecible que generase un recorte en el número de hospitales y camas, así como que la atención médica por servicios agudos se concentraría en 4 lugares y no en 10 como venía sucediendo previamente (generando problemas de sobrecapacidad). La generación de monopolios hospitalarios como consecuencia de búsqueda de la competitividad del modelo sueco ya ha sido documentada en la literatura. ${ }^{1,7}$

La mayor competencia e incentivos que se registraron durante la década de los 1990 derivaron en que los costos hospitalarios y las actividades se elevaran significativamente ${ }^{20}$ - entre un $6 \%$ y $8 \%$ anual. ${ }^{17} \mathrm{La}$ inclusión de proveedores privados de salud y la libertad de elección otorgada a los pacientes impulsó los gastos médicos hacia arriba. Ham \& Brommels ${ }^{13}$ señalaban que era una situación común que las estancias hospitalarias de algunos pacientes se extendieran mucho más de lo previsto debido a las pocas alternativas o facilidades de transferencia que poseían los pacientes dentro de un mismo condado. En estos casos, los condados se hacían cargo de todos los costos de esa estancia inapropiada hasta que el paciente podía llevarse a un hospital de tratamientos agudos.

De esta forma, las reformas en Suecia dejaron de funcionar en extenso. En 1995, Suecia regresó al sistema descentralizado previo a la reforma aunque la libre elección de los pacientes continuó. ${ }^{2}$ La experiencia de la reforma dejo que los principios básicos del modelo Stockholm fueron correctos, sin embargo, no se aplicaron de la manera adecuada. ${ }^{12}$ Hasta la fecha no se tiene documentado en Suecia que la calidad o equidad de la atención médica haya sido mejorada con la introducción de las reformas. Por ejemplo, no existe ninguna evidencia de que la reducción de los días de estancia hospitalaria haya reducido el número de reingresos o que la población adulta no haya sido discriminada. Asimismo, el problema de los tiempos de espera es un fenómeno persistente dentro del sistema de salud en Suecia. Aún cuando inicialmente la reforma sanitaria del país apuntó a reducir esta problemática, los resultados finales no fueron favorables. Para 1997, el 37\% de los pacientes tenían que esperar entre zero y un día por una consulta ambulatoria, el $9 \%$ entre dos a cuatro días, $27 \%$ entre cinco a ocho días y $27 \%$ más de ocho días. ${ }^{13}$ De cualquier forma, como señala Anell, ${ }^{2}$ los tiempos de espera no son los indicadores más adecuados si se quiere evaluar una reforma integral de un sistema de salud como fue el caso sueco.

En la actualidad muchos condados suecos se encuentran en números rojos. El déficit total en 1998 ascendía a los cinco billones de coronas suecas. La fusión de hospitales no ha tenido ningún éxito (entre 1995 y 1997 cerca de 40 hospitales públicos fueron fusionados en Suecia). Asimismo, existe una ausencia alarmante de enfermeras en muchos condados y el sector privado cada año gana mayor terreno. ${ }^{11,12}$

Nuevas reformas competitivas se han realizado en Suecia durante los últimos años aunque con poco éxito.

a Dahlström A, Ramström D. Stockholmsmodellen: efekter, problem, vägval: en sammanfattning av ett utvärderingsprogram. Stockholm: SPRI; 1995. (SPRI report, 398) 
En 1999, un hospital general de Estocolmo fue vendido a una compañía privada por el consejo del condado. La compañía mantiene un contrato con el gobierno y es en promedio un 7\%-12\% más barata si se le compara con otros hospitales públicos. El contrato de tres años fue extendido por otros cuatro años (hasta el 2006). En el año 2001, el 40\% de la atención primaria era otorgada por agentes privados; la participación de mercado global en el sistema sanitario sueco para el sector privado era de un 23\% para el área metropolitana de Estocolmo y del 7\% en zonas del interior del país. ${ }^{17}$ Sin embargo, a partir de ese mismo año, la privatización de hospitales públicos por privados ha sido fuertemente frenada por las autoridades suecas.

\section{CONCLUSIÓNES}

El nuevo sistema de salud sueco generado por medio de las reformas competitivas y de transferencia de costos no alcanzaron los objetivos propuestos. En un inició fue evidente las mejoras en la productividad global del sistema en aquellos condados donde se produjeron las reformas. Sin embargo, ello conllevó posteriormente a que dentro del sistema de salubridad sueco se elevaran posteriormente los costos, así como se concentraran los servicios de la atención médica en algunos servicios, con la consiguiente reducción de la satisfacción de los pacientes y la nula reducción de los tiempos de espera. 


\section{REFERENCIAS}

1. Anell A. The monopolistic integrated model and health care reform: the Swedish experience. Health Policy. 1996;37(1):19-33.

2. Anell A. Swedish healthcare under pressure. Health Econ. 2005;14(Suppl 1):S237-54.

3. Andersson K, Petzold MG, Sonesson C, Lönnroth K, Carlsten A. Do policy changes in the pharmaceutical reimbursement schedule affect drug expenditures? Interrupted time series analysis of cost, volume and cost per volume trends in Sweden 1986-2002. Health Policy. 2006;79(2-3):231-43.

4. Bergman SE. Swedish models of health care reform: a review and assessment. Int J Health Plann Manage. 1998;13(2):91-106.

5. Bruce A, Jonsson E. Competition in the provision of health care: the experience of the US, Sweden and Britain. Aldershot: Ashgate; 1996.

6. Carpentier C, Samuelson LA. Effekter av en sjukvårdsreform: Erfarenheter av Stockholmsmodellen. Sollentuna. (1999). Nerenius \& Santérus Förlag. apud Håkansson S. Productivity changes after introduction of prospective hospital payments in Sweden. Casemix Q. 2000;2(2):41-57.

7. Diderichsen F. Market reforms in health care and sustainability of the welfare state: lessons from Sweden. Health Policy 1995;32(1-3):141-53.

8. Enthoven AC. Management information and analysis for the Swedish health care system. Lund: Swedish Institute for Health Economics; 1989. (IHE working paper, 7)

9. Färe R, Grosskopf S, Lindgren B, Poullier JP. Productivity growth in hospital cost inflation 19851993. Med Care. 1997;35(4):354-66.

10. Gerdtham U, Rehnberg C. The impact of internal markets on health care efficiency: evidence from health care reforms in Sweden. Stockholm: Stockholm School of Economics, 1997. (Working paper, 170)

11. Håkansson S, Nordling S. Sweden. In: Raffel MW, editor. Health care and reform in industrialized countries. Pennsylvania: Pennsylvania University Press, 1997. p 250-82.

12. Håkansson S. Productivity changes after introduction of prospective hospital payments in Sweden. Casemix Q. 2000;2(2):41-57.

13. Ham C, Brommels M. Health care reforms in the Netherlands, Sweden and the United Kingdom. Health Aff (Millwood). 1994;13(5):106-19.
14. Hanning M. Maximum waiting-time guarantee - an attempt to reduce waiting lists in Sweden. Health Policy. 1996;36(1):17-35.

15. Harrison MI, Calltorp J. The reorientation of marketoriented reforms in Swedish health-care. Health Policy. 2000;50(3):219-40.

16. Hjortsberg C, Ghatnekar O. Health care systems in transition. Sweden: European Observatory on Health Care Systems, 2001.

17. Hjertqvist J. Meeting the challenges to European Healthcare. Lessons learned from the 'Stockholm Revolution'. Pharmacoeconomics. 2002;20(Suppl 3):47-53.

18. Jonsson E. Har betalning per patient givit mer vård för pengarna? En jämförande utvärdering för perioden 1986-1993. Stockholm: SPRI förmedlar, 1996; 4. apud Håkansson S. Productivity changes after introduction of prospective hospital payments in Sweden. Casemix Q. 2000;2(2):41-57.

19. Laugesen M. Why some market reforms lack legitimacy in health care. J Health Polit Policy Law. 2005;30(6):1065-100.

20. León S, Rico A. Sweden. In: European Observatory on Health Care Systems. Health care systems in eight countries: trends and challenges. London: School of Economics and Political Science Hub; 2002. p. 91-102.

21. Marmor TR, Mashaw JL Understanding social insurance: fairness, affordability, and the 'modernization' of Social Security and Medicare. Health Aff (Millwood). 2006;25(3):w114-34.

22. Martin JP. The experience of OECD countries in coping with rising health cost. Paris: Organization for Economic Cooperation and Development, 2003.

23. Mossialos E. Citizens' views on health care systems in the 15 member states of the European Union. Health Econ. 1997;6(2):109-16.

24. Programa de las Naciones Unidas para el Desarrollo. Informe sobre desarrollo humano 2004: la libertad cultural en el mundo diverso de hoy. Madrid: MundiPrensa, 2004.

25. Rosenthal M; Daniels N Beyond competition: the normative implications of consumer-driven health plans. J Health Polit Policy Law. 2006;31(3):671-85.

26. Shackley P, Healey A. Creating a market: an economic analysis of the purchaser-provider model. Health Policy. 1993;25(1-2):153-68.

27. World Health Organization (WHO). European health care reforms. Copenhagen; 1996. 